NSF-ITP-00-18

hep-th/0003251

\title{
Excision of 'repulson' singularities: a spacetime result and its gauge theory analogue
}

\author{
Amanda W. Peet巴 \\ Institute for Theoretical Physics, \\ University of California, \\ Santa Barbara, CA 93106-4030, U.S.A.
}

Based on [1] with C.V. Johnson and J. Polchinski

\begin{abstract}
We discuss spacetime singularity resolution in the context of the gravity / gauge correspondence, for brane systems which give rise to gauge theories with eight supercharges and no hypermultiplets. The discussion is aimed at non-experts. Writeup of talk on [1] for proceedings of PASCOS-99 (10 - 16 Dec 1999, Granlibakken, Lake Tahoe); also relevant to Way Beyond the Standard Models Conference (31 Jan - 5 Feb 2000, Aspen, Colorado) and CIAR Gravity and Cosmology Programme Meeting (19 - 22 Feb 2000, Banff, Canada).
\end{abstract}

\section{Introduction}

Spacetimes with singularities occur in many classical theories of gravity. This includes classical superstring theory i.e. supergravity, where there are examples of spacelike, null, and timelike singularities, some of which are naked. An important question is how, or indeed whether, quantum string theory resolves these singularities.

As pointed out in Ref. [2], some singularities are unphysical and cannot be patched up by string theory. A canonical example is the negative-mass Schwarzschild geometry which has a naked singularity. If string theory were to patch that up by smoothing it out to a small region of strong but finite curvature, then by mass negativity this would signal an instability of the theory as the vacuum would be unstable.

String theory resolves some spacetime singularities in a beautiful way. The basic idea is that once spacetime curvatures or other invariant measures of quantum corrections such as the dilaton are too large for supergravity to be applicable, other degrees of freedom take over.

\footnotetext{
${ }^{1}$ peet@itp.ucsb.edu
} 


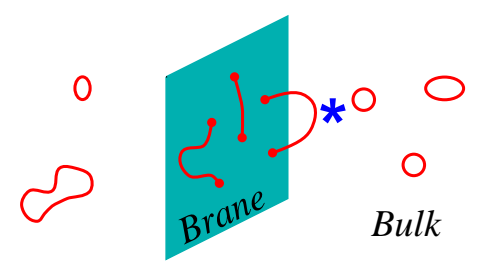

Figure 1: A D p-brane living in the $d=10$ bulk, with open and closed strings. The $\star$ represents open-closed interactions.

Examples of this are the gravity / gauge theory correspondences with sixteen supercharges of Ref. [3]. Let us now turn to an introduction to these correspondences; a comprehensive review of the subject including the original $A d S / \mathrm{CFT}$ correspondences of Ref. 体 may be found in Ref. [5].

The starting point for correspondences with sixteen supercharges is a system of $N \mathrm{D} p$ branes of Type II string theory. These $\mathrm{D} p$-branes are hypersurfaces where open strings must end. The open strings distinguish which brane they end on via their Chan-Paton factors, which they carry on their endpoints at zero energy cost. This gives rise to a $U(N)$ gauge theory living on the worldvolume of the $\mathrm{D} p$-branes. In addition, the $\mathrm{D} p$-branes carry mass and Ramond-Ramond charge in the ten-dimensional bulk due to interactions between open and closed strings. See Ref. [6] and Fig.1.

To engineer gravity/gauge theory correspondences, one takes a special low-energy limit of the $\mathrm{D} p$-brane system. In the limit, the complicated physics on the branes reduces to $d=p+1$ supersymmetric $U(N)$ Yang-Mills theory (SYM) with sixteen supercharges. For $p>3$, the SYM theory is nonrenormalizable and so there is a need for new ultraviolet degrees of freedom in order to define the theory. String theory provides them in a well understood fashion for $p<6$. We will not discuss the $p \geq 6$ cases here.

In the limit, gauge theory energies $E$ are taken to be well below the string scale, $E \ell_{\mathrm{s}} \rightarrow 0$. In order to keep the brane physics nontrivial, the gauge coupling on the branes, which is a derived quantity, $g_{\mathrm{SYM}}^{2(p)}=(2 \pi)^{p-2} g_{\mathrm{s}} \ell_{\mathrm{s}}^{p-3}$, is held fixed. Here $g_{\mathrm{s}}$ is the string coupling constant. BPS W-boson-like states in the gauge theory, which in the bulk picture are open strings stretched perpendicularly between $\mathrm{D} p$-branes, have mass $U \equiv r \ell_{\mathrm{s}}^{-2}$ which is also held fixed. In this relation, $r$ is the separation between the $\mathrm{D} p$-branes.

In string theory the bulk Newton constant is also a derived quantity, e.g. in $d=10$ we have $G_{10}=8 \pi^{6} g_{\mathrm{s}}^{2} \ell_{\mathrm{s}}^{8}$. The $\mathrm{D} p$-brane supergravity geometry may be written in terms of a harmonic function $H_{p}=1+c_{p} g_{\mathrm{s}} N\left(\ell_{\mathrm{s}} / r\right)^{7-p}$, where $c_{p}$ is a constant. Use of the above scaling relations results in the loss of the 1 from $H_{p}$, i.e. the asymptotically flat part of the geometry. As a result, the bulk description of the system of $N \mathrm{D} p$-branes in the above low-energy limit is in terms of string theory on the near-horizon geometry.

This limit is called the decoupling limit because at such low energies the coupling between open and closed strings is turned off. Hence, the gauge theory on the branes and the bulk string theory on the near-horizon geometry may each be considered as a unitary theory on their own. This property makes a duality between the two theories feasible. Such a duality is often termed 'holographic' because it relates a $d=10$ bulk string theory to a $d=p+1$ gauge field theory. Note also that in this limit, there is no gravity on the brane, unlike the 
situation that occurs in the Randall-Sundrum scenario of Ref.s [7].

For general $p$, the spacetime curvature of the $\mathrm{D} p$-brane near-horizon geometry is not constant but varies with radial variable $U$. The local value of the string coupling, $g_{\mathrm{s}} e^{\Phi}$, also varies, but differently. For example, the cases $p<3$ have a dilaton singularity at $U \rightarrow 0$ and a curvature singularity at $U \rightarrow \infty$, while for $p>3$ cases it is the other way around. We then need to know what degrees of freedom take over where the bulk $d=10$ supergravity description goes bad. In regions where the dilaton is strong, we use S-duality which takes us to a description with a weak dilaton. In regions where the curvatures become strong, it turns out that the right description is the SYM theory. For details, see Ref. [3] and further development reviewed in Ref. [8]. Before moving on, we will just note that by the nature of duality, only one description may be weakly coupled in any given region of parameter- and $U$ - space.

If we wish to do gauge theory at finite temperature $T$, we use the nonextremal D-brane geometry with Hawking temperature $T$, taken in the decoupling limit. This gives rise to a black hole type generalization of the near-horizon geometry. In principle, this is a useful picture in the context of the black hole information problem, because we have a duality of the black hole type system to a quantum field theory which is a manifestly unitary theory. The difficulty in solving the information problem in practice is the strong-weak nature of the duality: when the bulk picture is weakly coupled (calculable), the brane picture is strongly coupled and vice-versa.

There have been many further generalizations of the prototype correspondences; see Ref.s [5, 10]. We note here, however, that some attempts to construct supergravity duals to certain gauge theories turned out to be unphysical, partly because analyzing the supergravity theory in situations of interest is generally very difficult. See e.g. Ref. [9] for a discussion of some of the problems encountered.

\section{The pure $\mathcal{N}=2$ correspondence}

We are interested in a system which gives pure gauge theory with eight supercharges living on the branes. Examples with eight supercharges and lots of hypermultiplet matter included the original $A d S_{3} \times S^{3} \times T^{4}$ and $A d S_{3} \times S^{3} \times K 3$ correspondences and Ref.s [12]. However, we want no hypermultiplets, and this will have drastic consequences for the properties of our spacetime.

Our setup of Ref. [1] includes many dual realizations in terms of branes. The one on which we will concentrate is obtained by wrapping $\mathrm{D}(p+4)$-branes on $K 3$. Other options including $\mathrm{D}(p+1)$-branes strung between two parallel NS5-branes, and $\mathrm{D}(p+2)$ wrapped on collapsing $\mathrm{S}^{2}$ cycles in $K 3$. Related work appeared in yet another dual realization, the heterotic one, in Ref. [11.

\section{$2.1 \quad$ The setup}

Here we will focus on the $p=2$ case for simplicity. Our starting point is therefore a system of $N$ D6-branes wrapped on a $K 3$ surface. 
For the gauge theory side, we build the $d=2+1$ 't Hooft coupling out of the $d=6+1$ coupling and the $K 3$ volume $(2 \pi R)^{4}$ :

$$
\lambda_{2} \equiv g_{\mathrm{SYM}, 2}^{2} N=\frac{g_{\mathrm{SYM}, 6}^{2} N}{(2 \pi R)^{4}}=\frac{g_{\mathrm{s}} N \ell_{\mathrm{s}}^{3}}{R^{4}} .
$$

The gauge theory will indeed be $2+1$ dimensional as long as excitations are low-energy by comparison to the characteristic scale of excitations in the compact $K 3$. We therefore keep $1 / R$ finite in the decoupling limit.

For the bulk side of things, in constructing the supergravity solution we usually start by finding the conserved charges. Generally, a supergravity solution is then uniquely determined via a no-hair argument. It is important to realize, however, that this argument fails when the geometry has a naked singularity, and so we will have to proceed with caution if we encounter one.

We begin with $N$ D6-branes, so we have D6-brane charge $N$. Once we wrap a D6 on a $K 3$, an additional charge arises due to the curvature of $K 3$, see Ref.s [13]. As a result, we also have D2-brane charge $(-N)$. The ADM tension formula is protected by supersymmetry and so we have

$$
\tau_{\mathrm{ADM}}=\frac{N}{(2 \pi)^{3} g_{\mathrm{s}} \ell_{\mathrm{s}}^{7}} R^{4}-\frac{N}{(2 \pi)^{3} g_{\mathrm{s}} \ell_{\mathrm{s}}^{3}} .
$$

Applying the no-hair theorem naively, we find that the metric the string sees is, in the decoupling limit,

$$
\begin{aligned}
\frac{d S^{2}}{\ell_{\mathrm{s}}^{2}}= & \frac{R^{-2} d x_{\|}^{2}}{\sqrt{\left(\lambda_{2} / U\right)\left[1-\left(\lambda_{2} / U\right)\right]}}+\sqrt{\frac{\left[1-\left(\lambda_{2} / U\right)\right]}{\left(\lambda_{2} / U\right)}} d s_{K 3}^{2} \\
& +R^{2} \sqrt{\left(\lambda_{2} / U\right)\left[1-\left(\lambda_{2} / U\right)\right]}\left\{d U^{2}+U^{2} d \Omega_{2}^{2}\right\} .
\end{aligned}
$$

We have used spherical coordinates $\left(U, \Omega_{2}\right)$ for the three dimensions transverse to both the $K 3$ and the $d=2+1$ worldvolume $x_{\|}$.

Let us inspect our classical metric. At

$$
U=\lambda_{2}
$$

there is a pathology; some components of the metric become imaginary for $U<\lambda_{2}$. Computing curvature invariants, we find that there is a singularity at $U=\lambda_{2}$, the locus of which is a two-sphere. The would-be horizon, located where $g^{U U} \rightarrow 0$, is at $U=0$, and so the singularity is naked.

In a related (heterotic) context, this singularity was studied in Ref.s [14, 15], and in Ref. [15] it was dubbed the 'repulson' because massive particles are repelled by the singularity. This behavior is reminiscent of the inside of extremal Reissner-Nördstrom black hole.

Given our previous caveat on use of no-hair theorems, and the fact that some classical spacetime singularities are unphysical and cannot be resolved by quantum stringy effects, we have cause for concern about our repulson. In fact, it turns out that the repulson singularity is excised via a stringy mechanism, and we now turn to the description of this excision phenomenon. 


\subsection{Probe physics and spacetime singularity resolution}

The physics seen by a probe in string theory depends on the probe and the target. If we probe a target made of fundamental strings with another string, the best spatial resolution possible turns out to be the string scale $\ell_{\mathrm{s}}$. This happens because the string is an extended object; more energy-momentum pumped into the probe string does not result in greater positionspace resolution but rather in stretching the probe. The existence of a minimum distance is related to the T-duality symmetry of string theory (for a spatial direction compactified on a circle of radius $R$, T-duality exchanges $R / \ell_{\mathrm{s}} \leftrightarrow \ell_{\mathrm{S}} / R$ ). If a D-brane is used as a probe of other D-branes, different physics results. For example, in the case of D0-branes, Matrix Theory (see e.g. Ref. [16]) gives a characteristic scale of $g_{\mathrm{s}}^{1 / 3} \ell_{\mathrm{s}}$.

In our situation we are interested in probing the system of $N$ D6-branes wrapped on the $K 3$. It turns out that the best probe for answering our question about singularity resolution is a clone, namely a single D6-brane also wrapped on the $K 3$.

We begin with the spacetime or bulk side of the story. We take large- $N$, so that the probe D6 can be thought of as a 'test'-brane, and the $N$ 'source'-branes are represented by their supergravity solution. By supersymmetry, the static potential between the source and probe branes vanishes. The action for the probe brane turns out to be

$$
S=\int\left\{\frac{1}{2} \vec{v}^{2} \frac{R^{4}}{(2 \pi)^{2} g_{\mathrm{s}} \ell_{\mathrm{s}}^{7}}\left[1-2\left(\lambda_{2} / U\right)\right]\right\}+\mathcal{O}\left(\vec{v}^{4}\right),
$$

where $\vec{v}$ is the velocity of the brane in the $\left(U, \Omega_{2}\right)$ directions. The coefficient of $v^{i} v^{j}$ is the metric on moduli space, and we see that for the D-branes wrapped on $K 3$ it is not flat as it would have been for branes wrapped on $\mathrm{T}^{4}$. The moduli space metric has a zero, which signals the vanishing of the 'local' tension of the probe. The locus of this zero is a sphere of radius

$$
U_{\mathrm{e}} \equiv 2 \lambda_{2}=2 g_{\mathrm{SYM}}^{2} N
$$

Notice that the radius $U_{\mathrm{e}}$ is twice as far out as the radius of the repulson singularity. We may now ask what physics is signified by the vanishing of the local tension of the probe. By inspection of the metric and dilaton, we find that nothing special happens at $U_{\mathrm{e}}$. On the other hand, the volume of $K 3$, which varies with $U$ as

$$
\operatorname{Vol}(K 3)=\ell_{\mathrm{s}}^{4} \frac{\left[1-\left(\lambda_{2} / U\right)\right]}{\left(\lambda_{2} / U\right)}
$$

goes to the special value $\ell_{\mathrm{s}}^{4}$ at $U_{\mathrm{e}}$.

It is perhaps easiest to interpret the physics by performing $\mathrm{T}$ - and $\mathrm{S}$-dualities to turn the D6-brane wrapped on a $K 3$ into a heterotic string wrapped on a circle. The D6- and D2-brane charges $(+1,-1)$ turn into winding and momentum charges $(+1,-1)$, and the $\ell_{\mathrm{s}}^{4}$ sized $K 3$ turns into a $\ell_{\mathrm{s}}$-sized circle. From perturbative string theory it is known that such strings wound on such a circle are massless and provide the gauge bosons for an enhanced $S U(2)$ symmetry. Alternatively, in the dual realization with D3-branes strung between two NS5-branes, the $S U(2)$ is that of the two NS5-branes, which is restored at the radius $U_{\mathrm{e}}$

because brane bending due to the D3's causes the NS5's to touch there. Dualizing back to our original system, we still have the $S U(2)$ enhanced symmetry, at the locus $U=U_{\mathrm{e}}$, which 
we dub the 'enhançon'. This $S U(2)$ symmetry is broken at any distance $U>U_{\mathrm{e}}$; this is a Higgs mechanism in disguise.

We can also use the heterotic dual picture to understand physically why there is no notion of 'inside the enhançon' for the probe brane; it is just the minimum-distance phenomenon we mentioned at the beginning of this section. We can also compute the Compton wavelength of the probe as it approaches the enhançon, and we find that it expands smoothly upon approach to the enhançon locus. We refer the reader to Ref. [1] for details and further explanation.

Now let us imagine trying to build the singular repulson geometry, one brane at a time. Notice that the radius of the enhançon locus, $U_{\mathrm{e}}$ of Eq. (6), is linear in $N$. Thus, the first brane has a small enhançon radius. The second brane cannot go inside the enhançon radius of first brane, and so we get a pair of branes at finite separation. The third brane cannot go inside the enhançon radius of the first two, and so on; in this way a sphere of $N$ evenly spaced branes is built up. The source branes then form a 'Dyson sphere' of a radius twice that at which the classical naked singularity occurred. Therefore, the singularity is excised in quantum string theory it was never really there. The gravitational field is flat inside the Dyson sphere, by symmetry. Notice also that at large 't Hooft coupling this Dyson sphere is macroscopically large.

\subsection{The enhançon phenomenon and Seiberg-Witten theory}

In the last subsection we saw that stringy effects saved the classical spacetime from embarrassment by excising its naked singularity. We now proceed to exhibit the corresponding phenomenon on the gauge theory side of the story.

For the analogue of our singularity excision in classical gravity, we must look to nonperturbative gauge theory. Fortunately, all we need for study of the moduli space physics is the Seiberg-Witten ( $\mathrm{S}-\mathrm{W})$ curve. For the case $N=2$, in moduli space there are two branch points possessing enhanced gauge symmetry, namely where the monopole and dyon of the theory become massless. The large- $N$ version of the SW curve] was worked out in Ref. [17],

$$
y^{2}=\prod_{i=1}^{N}\left(x-\phi_{i}\right)^{2}-\Lambda^{2 N},
$$

where $\Lambda$ is a nonperturbatively generated scale and the $\phi_{i}$ are the adjoint scalar field vevs.

In the situation of interest, namely the system of a single probe brane far away from $(N-1)$ other branes, the vevs are

$$
\phi_{i}=0, i=1 \ldots N-1, \quad \phi_{N} \gg \Lambda .
$$

Solving for the branch points $y=0$ gives $2(N-1)$ points $x$ on an $S^{1}$ of radius $\Lambda$, and two at $x=\phi_{N}($ to $\mathcal{O}(1 / N))$. More generally, little is known about Seiberg-Witten theory for the $d=p+1$ gauge theories, but by analogy we will obtain a $S^{4-p}$ of radius $\Lambda$. This gives the brane positions as shown in Fig.2.

We can also use the $\mathrm{S}-\mathrm{W}$ curve to deduce the physics if we try to bring the probe inside the sphere. For this, we set $\phi_{N}<\Lambda$. By solving for the branch points in this case, we find

\footnotetext{
${ }^{2}$ We have switched to the $p=3$ case for convenience.
} 


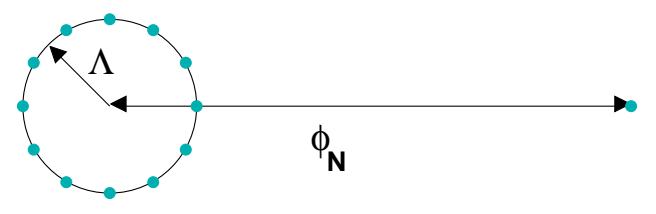

Figure 2: The brane positions as obtained by studying the Seiberg-Witten curve.

that they all lie on the sphere. The conclusion we can draw about the physics is that as we try to adiabatically move the probe brane 'inside' the sphere, it actually smoothly melts into the sphere.

It is worth noting at this point that there are other solutions for the branch points, but we used a symmetry argument by analogy with the bulk computation to pin down the spherically symmetric one. This suggests that there are other configurations on the bulk side which correspond to the other solutions from the SW curve, or that there is a degeneracy to explore.

Although we do not have space to discuss the details here, we have constructed the phase diagram of the $d=p+1$ systems with eight supercharges. This turns out to be significantly less straightforward than for sixteen supercharges. One reason is that there is a region of the phase diagram which does not have an obvious weakly coupled dual. This is the region describing energies of the order of the masses of strings stretched between different branes on the enhançon sphere. In addition, the finite temperature version of our setup does not permit black hole horizons. For the details, and some suggestions about the nature of the missing component of the phase diagram, we refer the reader to Ref. [1].

Ref. [18] shows that the enhançon phenomenon also appears in $S O(2 N+1), U S p(2 N)$ and $S O(2 N)$ gauge theories.

In the future we expect to develop further the physics of the enhançon phenomenon, and to make links with other recent studies of singularity resolution in string theory, such as Ref. [9] and Ref. [19].

\section{Acknowledgments}

The author wishes to acknowledge co-authors on [1], and in addition helpful discussions with Eric D'Hoker and Gary Horowitz.

This work was supported in part by NSF grant PHY94-07194. 


\section{References}

[1] C.V. Johnson, A.W. Peet and J. Polchinski, hep-th/9911161, to be published in Phys. Rev. D15 (April 2000).

[2] G.T. Horowitz and R.C. Myers, Gen. Rel. Grav. 27, 915 (1995) gr-qc/9503062.

[3] N. Itzhaki, J. Maldacena, J. Sonnenschein and S. Yankielowicz, Phys. Rev. D58, 046004 (1998) hep-th/9802042.

[4] J. Maldacena, Adv. Theor. Math. Phys. 2, 231 (1998) hep-th/9711200.

[5] O. Aharony, S.S. Gubser, J. Maldacena, H. Ooguri and Y. Oz, Phys. Rept. 323, 183 (2000) hep-th/9905111.

[6] J. Polchinski, String Theory, Cambridge University Press 1997.

[7] L. Randall and R. Sundrum, Phys. Rev. Lett. 83, 3370 (1999) [hep-ph/9905221; Phys. Rev. Lett. 83, 4690 (1999) hep-th/9906064.

[8] E. J. Martinec, hep-th/9909049.

[9] S.S. Gubser, hep-th/0002160.

[10] A. Giveon and D. Kutasov, JHEP 9910, 034 (1999) hep-th/9909110.

[11] M. Krogh, JHEP 9912, 018 (1999) hep-th/9911084.

[12] I.R. Klebanov and N.A. Nekrasov, hep-th/9911096; I.R. Klebanov and A.A. Tseytlin, hep-th/0002159.

[13] K. Dasgupta, D.P. Jatkar and S. Mukhi, Nucl. Phys. B523, 465 (1998) hep-

th/9707224; C.P. Bachas, P. Bain and M.B. Green, JHEP 05, 011 (1999) hepth/9903210].

[14] K. Behrndt, Nucl. Phys. B455, 188 (1995) hep-th/9506106.

[15] R.E. Kallosh and A.D. Linde, Phys. Rev. D52, 7137 (1995) hep-th/9507022.

[16] T. Banks, hep-th/9911068.

[17] P.C. Argyres and A.E. Faraggi, Phys. Rev. Lett. 74, 3931 (1995) hep-th/9411057; A. Klemm, W. Lerche, S. Yankielowicz and S. Theisen, Phys. Lett. B344, 169 (1995) hep-th/9411048.

[18] L. Jarv and C.V. Johnson, hep-th/0002244.

[19] J. Polchinski and M. J. Strassler, hep-th/0003136. 